Check for updates

Cite this: RSC Adv., 2018, 8, 4221

Received 30th September 2017 Accepted 31st December 2017

DOI: $10.1039 / c 7 r a 10824 a$

rsc.li/rsc-advances

\section{Design of bright near-infrared-emitting quantum dots capped with different stabilizing ligands for tumor targeting $\dagger$}

\author{
Xijing Liu, (D) a Peijiang Zhou, ${ }^{* a}$ Hongyu Liu, ${ }^{a}$ Hongju Zhan, ${ }^{b}$ Qiang Zhang, ${ }^{c}$ \\ Yanan Zhao ${ }^{c}$ and Yun Chen (iD *c
}

In this study, a facile formulation of near-infrared (NIR)-emitting $\mathrm{CdHgTe} / \mathrm{CdS} / \mathrm{CdZnS}$ quantum dots (QDs) was fabricated and coated with three different thiol ligands, N-acetyl-L-cysteine (NAC), 3-mercaptopropionic acid (MPA) and thioglycolic acid (TGA). The optical properties, morphology, hydrodynamic diameters, structure and surface chemistries of the developed QDs were comprehensively assessed. As a therapeutic drug, NAC provides excellent biocompatibility and anti-oxidant activity both in vitro and in vivo. Given their core-shell-shell structure, the QDs induced no toxicity in living bodies even at a concentration of $20 \mathrm{mg} \mathrm{kg}$. Compared with NAC-capped QDs, TGA- and MPA-capped QDs demonstrated poor tumor-targeting capabilities because of their larger hydrodynamic diameters and inferior biocompatibility. Furthermore, the use of different stabilizing ligands resulted in various major changes in organ biodistribution and clearance from the body. In terms of rapid metabolization through renal clearance, the NAC-capped core-shell-shell QDs will be an alternative probe in cellular metabolism studies and image-guided surgery for tumor targeting and removal.

\section{Introduction}

Cancer is a type of pervasive disease that affects the entire human body. Fortunately, scientists can provide exciting approaches to fight even the most aggressive forms of cancer. ${ }^{1}$ However, several forms of cancer do not respond well to these therapies as the tumor returns even after the best chemotherapy treatments or an onslaught of drugs. ${ }^{\mathbf{1 , 2}}$ Currently, researchers recognize that there is an incredible opportunity to save lives through the early detection of cancer. ${ }^{1-3}$ The problem remains that many tumor diagnosis tools are costly, invasive and most importantly incapable of detecting small tumors (with a size under $1 \mathrm{~cm}) .{ }^{4,5}$ In this case, it is significant that high-resolution imaging by NIR-emitting QDs could be a powerful tool for the early detection of cancer. The diagnostic window (NIR region of the spectrum, 700-900 $\mathrm{nm}$ ) can be explored for in vivo deeptissue imaging because some major chromophores (haemoglobin and water) of the living body have a local absorption minimum in that region. ${ }^{6,7}$ By virtue of the leaky nature of

\footnotetext{
${ }^{a}$ School of Resource and Environmental Science, Hubei Biomass-Resource Chemistry and Environmental Biotechnology Key Laboratory, Wuhan University, Wuhan 430079, China.E-mail: zhoupj@whu.edu.cn; 2013102050031@whu.edu.cn

${ }^{b}$ Jingchu University of Technology, Jingmen 448000, China

${ }^{c}$ Department of Biomedical Engineering, School of Basic Medical Sciences, Wuhan University, Wuhan 430071, China.E-mail: yunchen@whu.edu.cn

$\dagger$ Electronic supplementary information (ESI) available. See DOI: 10.1039/c7ra10824a
}

tumor blood vessels, high quality QDs can excellently accumulate within tumors through the enhanced permeability and retention (EPR) effect. ${ }^{8}$

Alloyed CdHgTe is the most attractive material among NIRemitting nanocrystals because of its high crystallinity, strong fluorescence and wide range of tunable optical properties., ${ }^{\mathbf{9}, 10}$ However, the continuous growth of CdHgTe results in an inhomogeneous composition and poor optical stability. ${ }^{9}$ The optical quality of CdHgTe QDs can be improved by adding an overlayer of epitaxially grown CdS or ZnS. Moreover, owing to the existence of strain-induced defects, problems (such as low PLQYs, broad full width at half maximum (FWHM) and large sizes) will arise when assurance the thickness of shells by the routine synthesis method. ${ }^{\mathbf{1 1}}$ Thus, we report a facile one-pot method for fabricating NIR-emitting CdHgTe/CdS/CdZnS core-shell-shell (CSS) QDs with ultra-small sizes. Unreacted free $\mathrm{Cd}$ ions were present due to the high Cd-to-Te molar ratio, which supplied a steady Cd source. At high temperature, the free $\mathrm{Cd}$ ions gradually reacted with the decomposition products of the thiol ligands, consequently forming a CdS shell around the CdHgTe core seeds. ${ }^{12}$ Another CdZnS shell was then grown directly on the surface of the CdHgTe/CdS QDs. This double shell structure effectively confined the core region and separated it from the surface, thereby successfully improving the PLQYs and stability.

It has been about 40 years since the notion of targeted nanoparticle was introduced, but few materials have reached clinical trials. ${ }^{13}$ The main reason for this phenomenon is 
attributed to the disadvantages presented by the materials, such as low PLQYs, instability and potential toxicity. ${ }^{\mathbf{1 4 , 1 5}}$ Up to now, the TOP/TOPO synthetic approach was one of the most mature methods for preparing highly fluorescent QDs. ${ }^{16}$ However, the use of the highly flammable and toxic dimethyl cadmium limited the applicability as bio-labeling materials. ${ }^{17}$ For QDs that are prefabricated in an aqueous phase, 3-mercaptopropionic acid (MPA) and thioglycolic acid (TGA) are the most commonly used stabilizer. ${ }^{17,18}$ Unfortunately, both ligands are carcinogenic, and the generated reactive oxygen species (ROS) are considered as one of the most important causes of cytotoxicity of QDs. ${ }^{10,19,20}$ Therefore, a functionalization strategy must be developed to mitigate the oxidative stress and QDinduced cytotoxicity. As a precursor of intracellular cysteine and glutathione, $N$-acetyl-L-cysteine (NAC) is readily deacetylated in cells to promote intracellular glutathione synthesis. Thus, this antidote induces numerous protective effects against DNA damage and carcinogenesis, which are related to its nucleophilicity, modulation of metabolism and antiinflammatory activity. ${ }^{\mathbf{1 0 , 1 9 , 2 0}}$ We subsequently assessed the influence of the three abovementioned ligands on the growth kinetics, optical properties, surface chemistry and toxicity of the QDs. Although the TGA- and MPA-capped QDs offered faster shifts to long emission wavelengths, NAC-capped QDs presented better biocompatibility and resistance to oxidation.

There is no optimal size or surface charge for in vivo tumor targeting because of the high degree of tumor-to-tumor variability. ${ }^{\mathbf{1 4 2 1}}$ Typically, spherical QDs with an hydrodynamic diameter (HD) of less than $100 \mathrm{~nm}$ have been demonstrated as effective in tumor penetration. ${ }^{\mathbf{1 4}}$ However, their clinical application has been hindered by concerns over the toxicity of their heavy metal constituents. ${ }^{2,6}$ The strategy for minimizing toxicity is to completely remove the QDs from the body in a reasonable amount of time. ${ }^{22,23}$ Although hepatic clearance is unpredictable and variable, Choi et al. prescribed an HD of approximately $5.5 \mathrm{~nm}$ for the renal filtration and urinary excretion of the QDs. $^{14,22,24}$ To date, there have been few cases of QDs that can target the tumor effectively and be excreted via renal clearance. This result means that the clearance of large-size QDs is quite slow, suggesting that the toxicity of QDs must be further investigated to determine the utility of QDs for targeting and therapy.

\section{Experimental section}

\section{Chemicals}

Cadmium chloride hemipentahydrate $\left(\mathrm{CdCl}_{2} \cdot 2.5 \mathrm{H}_{2} \mathrm{O}, 99 \%\right)$, mercury chloride $\left(\mathrm{HgCl}_{2}, 99 \%\right), \mathrm{N}$-acetyl-L-cysteine (NAC, 99\%), 3mercaptopropionic acid (MPA, 99\%), thioglycolic acid (TGA, 97\%), tellurium powder (Te, 99.9\%), potassium borohydride $\left(\mathrm{KBH}_{4}\right.$, $99 \%)$, zinc acetate dehydrate $\left(\mathrm{Zn}(\mathrm{Ac})_{2} \cdot 2 \mathrm{H}_{2} \mathrm{O}, 98 \%\right)$, sodium sulfide $\left(\mathrm{Na}_{2} \mathrm{~S}, 99 \%\right)$, bovine serum albumin (BSA, 99\%), phosphate buffer saline (PBS, $\mathrm{pH}=7.2$ ), Dulbecco's modified Eagle's medium (DMEM), 3-(4,5-dimethylthialzol-2-yl)-2,5-diphenyltetrazolium bromide (MTT), reagent grade ethanol and isopropanol were purchased from Sigma Aldrich. Sodium hydroxide ( $\mathrm{NaOH}, 99 \%)$ and hydrogen peroxide $\left(\mathrm{H}_{2} \mathrm{O}_{2}, 30 \%\right.$ in water) were purchased from Sinopharm Chemical Regent Co., Ltd.

\section{Device}

The synthesis of QDs was carried out on a microwave digestion/ extraction system (Milestone, Italy). The system operates at 2450 $\mathrm{MHz}$ and 0-1600 W. Exclusive Teflon ${ }^{\circledR}$ inner vessels with a volume of $50 \mathrm{~mL}$ are also equipped to provide security in reactions that require a high temperature $\left(300{ }^{\circ} \mathrm{C}\right)$ and pressure (10 MPa).

\section{Synthesis of thiol-capped CdHgTe/CdS/CdZnS QDs}

NaHTe was synthesized according to a procedure published elsewhere. ${ }^{18}$ Briefly, $160 \mathrm{mg}$ of Te powder were reduced by excess potassium borohydride in a $20 \mathrm{~mL}$ three-neck flask under nitrogen flow at room temperature. After one hour, the black Te powder disappeared, and a clear solution appeared. $0.5 \mathrm{M} \mathrm{H}_{2} \mathrm{SO}_{4}$ was slowly introduced to generate $\mathrm{H}_{2}$ Te gas that was discharged by nitrogen flow into another flask containing $100 \mathrm{~mL}$ of $0.01 \mathrm{M} \mathrm{NaOH}$ aqueous solution. For all samples, the CdTe precursor solution was obtained by adding NaHTe solution to $\mathrm{N}_{2}$-saturated $\mathrm{CdCl}_{2}$ solution at $\mathrm{pH} 8.5$ in the presence of thiol. The solution changed immediately from colorless to orange. Then, an appropriate amount of $\mathrm{Hg}^{2+}$ aqueous solution, prepared by an $\mathrm{N}_{2}$-saturated aqueous solution of $\mathrm{HgCl}_{2}$, was added to the CdTe precursor solution. The mixture was stirred for one hour, during which the solution turned to dark brown. The initial molar ratio of $\mathrm{Cd}^{2+}: \mathrm{Hg}^{2+}:$ thiol $: \mathrm{Te}^{2-}$ was set to $1: 0.05: 1.9: 0.2$, whereas the concentration of the precursors was $1.25 \mathrm{mM}$ with reference to the concentration of $\mathrm{Cd}^{2+} .20 \mathrm{~mL}$ of the $\mathrm{CdHgTe} / \mathrm{CdS}$ precursor solution was injected into the $50 \mathrm{~mL}$ Teflon inner vessel and heated inside the microwave digestion furnace for a given time and at a specific temperature. The temperature and time for the first process were $80^{\circ} \mathrm{C}$ and $60 \mathrm{~s}$, respectively. A relatively high temperature $\left(120^{\circ} \mathrm{C}\right)$ was selected for the second process as well as a duration of $10 \mathrm{~min} .^{12}$ After cooling to $<50{ }^{\circ} \mathrm{C}$ naturally, $\left.0.14 \mathrm{~mL} \mathrm{Zn(Ac)}\right)_{2}$ solution $(0.10$ $\mathrm{M})$ and $0.25 \mathrm{~mL} \mathrm{Na}_{2} \mathrm{~S}$ solution $(0.10 \mathrm{M})$ were dissolved in the crude solution, and either NAC, MPA or TGA $(0.10 \mathrm{M})$ was added under a protective stream of nitrogen. The molar ratio of $\mathrm{Zn}^{2+}$ : thiol was set to $1: 2$. The $\mathrm{pH}$ was adjusted to $8.5 \mathrm{using}$ 1.0 $\mathrm{M} \mathrm{NaOH}$ solution, and the solution was irradiated with microwaves. The prepared CdHgTe/CdS/CdZnS QD solution was purified by centrifugation 3 times with isopropanol and then dried in a freeze drier (Christ Alpha 1-2 LD, Germany) for $24 \mathrm{~h}$.

\section{Characterization}

UV-Vis and PL spectra were obtained on a Shimadzu UV-1601 Spectrophotometer and a FS 5-NIR fluorescence spectrophotometer, respectively. The room-temperature PLQYs were estimated using CY7 (PLQY $=28 \%$ ) in ethanol which was freshly prepared with the aim to reduce the measurement error. ${ }^{17}$ All absorption and fluorescence measurements were carried out at room temperature under ambient conditions. Transmission Electron Microscopy (TEM) and high-resolution TEM (HRTEM) images were obtained on a JEOL model JEM 2100 with an acceleration voltage of $200 \mathrm{kV}$. The samples were prepared by drop-coating the dilute solution onto ultrathin carbon film- 
supported copper grids and evaporating the excess solvent. Light-scattering analysis was performed using a Malvern Zetasizer Nano ZEN3700 dynamic light scatter (DLS). Powder X-ray diffraction (XRD) patterns were acquired using a Bruker-Axs D8 advanced diffractometer with $\mathrm{Cu} \mathrm{K} \alpha$ radiation. X-ray photoelectron spectroscopy (XPS) measurements were performed with a Kratos XSAM-800 apparatus using a monochromic Mg K $\alpha$ source at $1253.6 \mathrm{eV}$. Fourier Transform Infrared Spectroscopy (FT-IR) was obtained on a NICOLET 5700 FT-IR spectrometer through potassium bromide tabletting. Fluorescence intensity decay curves were plotted using an FL 900 single-photon counting system (Edinburgh Instrument). The excitation wavelength was $440 \mathrm{~nm}$. The recorded decay curves were fitted with a multiexponential function deconvoluted with the system response.

\section{In vitro experiments}

Fixed Hela cells were used to investigate the photostability of the as-prepared QDs. Briefly, 5000 cells were fixed with ethanol $(1 \mathrm{~mL}, 75 \%)$ and washed with PBS three times. Thereafter, the cells were incubated in 1\% BSA solution in PBS for $30 \mathrm{~min}$ at $37^{\circ} \mathrm{C}$ and then in $20 \mu \mathrm{L} \mathrm{CdHgTe} / \mathrm{CdS} / \mathrm{CdZnS}$ QDs $(0.8 \mathrm{mM})$ for $20 \mathrm{~min}$ at $37^{\circ} \mathrm{C}$. After that, the cells were carefully washed with PBS three times to remove the unbound QDs (QD concentrations were calculated following a method previously published by $\mathrm{Yu}$ et $a{ }^{25}$ ). The fixed cells stained with the $\mathrm{CdHgTe} / \mathrm{CdS} /$ CdZnS QDs were continuously irradiated by UV excitation. For live Hela cells imaging, the cells were propagated in Dulbecco's Modified Eagle's Medium (DMEM) supplemented with fetal blood serum (FBS, 10\%). Then, the cultured cells were trypsinized and resuspended in DMEM medium. After $24 \mathrm{~h}$ of incubation at $37^{\circ} \mathrm{C}$ in a humidified atmosphere with $5 \% \mathrm{CO}_{2}$ (about $80 \%$ confluence), the cells were rinsed with PBS three times; then, serum-free DMEM medium containing $4 \mathrm{nmol} \mathrm{mL}^{-1}$ $\mathrm{CdHgTe} / \mathrm{CdS} / \mathrm{CdZnS}$ QDs were added and incubated for $24 \mathrm{~h}$. The stained cells were carefully rinsed three times with PBS to remove the unbound QDs before all measurements. The fluorescent imaging of the stained Hela cells was conducted on an Olympus IX71 inverted fluorescence microscope (Olympus Optical Co.).

\section{In vivo toxicity evaluation}

BALB/c nude mice (6-7 weeks old) were obtained from the Animal Biosafety Level-III Laboratory at the Animal Experiment Center of Wuhan University. All experiments were performed in accordance with the standards of the National Institute of Health guide for the care and use of laboratory animals and were approved by the Institutional Animal Care and Use Committee (IACUC) of the Center for Animal Experiment, Wuhan University (Wuhan, China). The mice were acclimated to the animal facility for at least $72 \mathrm{~h}$ prior to experimentation. Then, all mice were randomized into test groups and a control group. $20 \mathrm{mg} \mathrm{kg}^{-1}$ of $\mathrm{CdHgTe} / \mathrm{CdS} / \mathrm{CdZnS}$ QDs was administered intravenously into the mice of the test groups. The control group was injected with normal saline solution under the same conditions. After 14 days of monitoring, blood and tissues were harvested for analysis. Hematological data were analyzed with a Mindray BC-2800Vet Auto Hematology Analyzer. Biochemical data were analyzed with a Fuji Dri-Chem Clinical Chemistry Analyzer FDC 3500 . The tissues were fixed in $10 \%$ formalin, embedded in paraffin, sectioned, and stained with hematoxylin and eosin. The images were obtained using an Olympus IX71 inverted fluorescence microscope (Olympus Optical Co.). The mice were sacrificed under anesthesia by injection of pentobarbital sodium through exsanguinations treatment. The bodies were lawfully disposed by the School of Basic Medical Sciences. We express our gratitude to all the animals that were sacrificed for science.

\section{Fluorescence imaging of tumor-bearing mice}

To establish tumor xenografts, $3 \times 10^{6}$ cells $/ 0.1 \mathrm{~mL}$ of Hela cells were injected subcutaneously into BALB/c 6-7 weeks old mice. One or two weeks after the tumor cell inoculation, the subcutaneous tumor mass grew to $0.5 \mathrm{~cm}$ in diameter, and the mice were ready for the in vivo imaging study detailed below. Then, the $\mathrm{CdHgTe} / \mathrm{CdS} / \mathrm{CdZnS}$ QDs $\left(10 \mathrm{mg} \mathrm{kg}^{-1}\right)$ were intravenously injected into the tail vein of the mice after they were placed under anesthesia by injection of pentobarbital sodium. After the injection of QDs, the mice were sacrificed under anesthesia for $8 \mathrm{~h}$ through exsanguination treatments. The heart, liver, spleen, lung and kidney were carefully collected and removed for ex vivo imaging. All the in vivo images were taken with a Bruker Xtreme BI in vivo imaging system (USA) with excitation at $455 \mathrm{~nm}$ and emission at $700-900 \mathrm{~nm}$.

\section{Results and discussion}

\section{Synthesis of differently stabilized CdHgTe/CdS/CdZnS QDs}

Fig. 1a displays the normalized ultraviolet photoluminescence (UV-PL) spectra of the CdHgTe/CdS/CdZnS QDs with different thiol ligands. The optical quality of the QDs depended on key synthetic parameters such as reaction time, reaction temperature, molar ratio of reactants in the original solution and $\mathrm{pH}$ value (Fig. S1†). All key parameters were kept constant to ensure

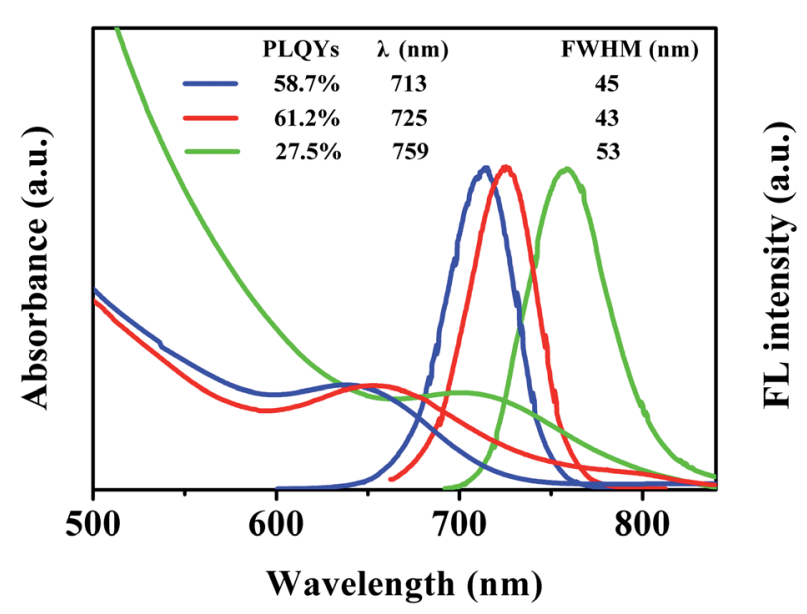

Fig. 1 UV-PL spectra of CdHgTe/CdS/CdZnS QDs capped by NAC (blue), MPA (red) and TGA (green). 
identical growth conditions for comparison purposes (Fig. 1). It is obvious that the PL emission range of the QDs is dependent on not only the structure (Fig. S1 $\dagger$ ), but also the surface state of the as-prepared QDs. ${ }^{26}$ The different thiol ligands resulted in varied growth mechanisms for the different chemical structures (Fig. S2 $\dagger$ ). ${ }^{27}$ As shown in Fig. 1, the TGA-capped CdHgTe/CdS/ CdZnS QDs shifted to the longest emission wavelengths in the set time. However, broad size distributions and low PLQYs limit the applicability of these QDs for biological application. After longer reaction times, MPA-capped QDs could reach the same emission wavelengths (Fig. S1†). The high PLQYs implied that the thermal decomposition of MPA effectively reduced the number of defects on the surface of the QDs. ${ }^{12}$ For NAC, the emission maxima failed to exceed $750 \mathrm{~nm}$ even after a long reaction time (Fig. S1 $\dagger$ ). Moreover, the excess energy caused non-radiative surface defects, broad size distributions and low PLQYs. ${ }^{12,28}$

\section{Characterization of CdHgTe/CdS QDs and CdHgTe/CdS/ CdZnS QDs}

Transmission electron microscopy (TEM) and high-resolution TEM (HRTEM) were employed to characterize the morphology of the as-prepared QDs. As seen from parts (a) to (c) of Fig. 2, the CSS QDs with three thiol ligands exhibited spherical shapes with homogeneous size distributions. For ternary systems such as CdHgTe, it is very challenging to obtain regular spherical shapes. ${ }^{9,29}$ However, the growth of the CdS and CdZnS shells around the CdHgTe core controlled the structure of the QDs. ${ }^{30,31}$ The HRTEM images verified the highly crystalline structure of the QDs. An interfacial layer was not observed between the core and the shell materials because of the high lattice match, thus indicating that the shell growth did not disturb the crystalline form of the core. ${ }^{31}$ Notably, the particle size of the TGA-capped QDs was larger than that of the MPA- or NAC-capped QDs, thereby implying that TGA provided the fastest growth kinetics for $\mathrm{CdHgTe} / \mathrm{CdS} / \mathrm{CdZnS} \mathrm{QDs}^{27}$ Size histograms of the asprepared QDs are shown in Fig. S3. $\dagger$ The average size and standard deviation of the particles were determined to be $2.1 \pm$ 0.6, $2.35 \pm 0.6$ and $2.65 \pm 0.9 \mathrm{~nm}$ for the $\mathrm{CdHgTe} / \mathrm{CdS} / \mathrm{CdZnS}$

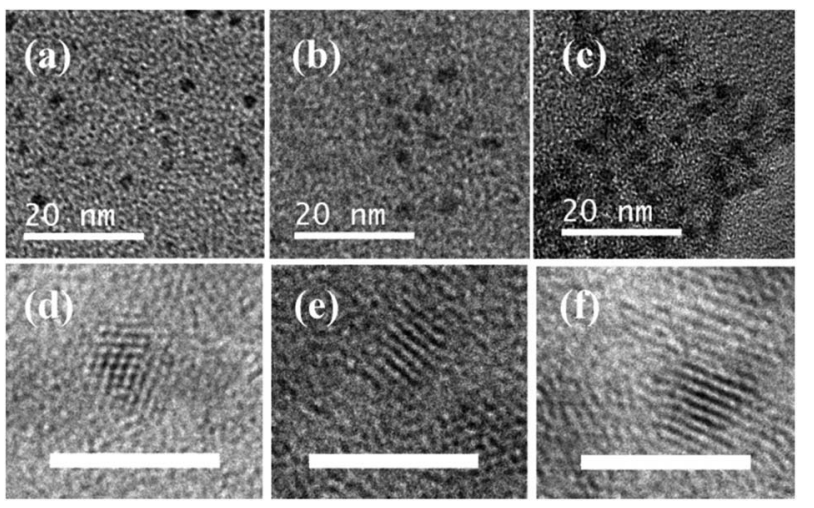

Fig. 2 TEM (top) and HRTEM (bottom) images of CdHgTe/CdS/CdZnS QDs capped by NAC (a, d), MPA (b, e) and TGA (c, f). Scale bars are $20 \mathrm{~nm}$ (TEM) and $5 \mathrm{~nm}$ (HRTEM).
QDs capped by NAC, MPA and TGA, respectively. This data agrees well with the FWHM theory in Fig. 1. ${ }^{30,31}$

Dynamic light scattering (DLS) was used to measure the hydrodynamic diameter (HD) of the QDs (Fig. 3a). The difference in the diameters measured by DLS and TEM is attributed to the different hydrophilic ligand molecules of the samples. Typically, the results of DLS represent the true diameters of the QDs. ${ }^{17}$ TEM could hardly detect the ligand molecules (e.g. NAC, MPA and TGA) due to their extremely low contrast. ${ }^{17}$ It is worth noting that the DLS size of the TGA-capped QDs $(>8.5 \mathrm{~nm})$ was distinctly larger than that measured by TEM $(2.65 \pm 0.9 \mathrm{~nm})$, thus showing that TGA was more easily degraded to bind on the surface atoms of the QDs. ${ }^{27}$

The powder X-ray diffraction (XRD) patterns of CdHgTe/CdS/ CdZnS QDs with different ligands are presented in Fig. 2b. For comparison, the standard XRD patterns of cubic HgTe, CdTe, CdS and ZnS are also shown (JCPDS no. 32-0665, 15-0770, 652887, 36-1450). ${ }^{29,32,33}$ All of the diffraction peaks lie between those inherent to the CdS and ZnS cubic phase. Notably, no distinct peak was observed from CdS or $\mathrm{ZnS}$, indicating the formation of a CdZnS shell on the surface of CdHgTe/CdS QDs. ${ }^{29,31}$

$\mathrm{X}$-ray photoelectron spectroscopy (XPS) was used to examine the chemical composition of the materials. As expected, the elements $\mathrm{Cd}, \mathrm{Te}, \mathrm{Hg}, \mathrm{S}, \mathrm{O}, \mathrm{C}$ and $\mathrm{Zn}$ were all detected in the survey spectra of the CdHgTe/CdS/CdZnS QDs (Fig. 3c). Notably, the typical peaks of N1s at $398.4 \mathrm{eV}$ and $\mathrm{N}$ loss at $424.5 \mathrm{eV}$ appeared in the spectrum of the NAC-capped QDs. This finding demonstrated that the acetyl amino groups combined during the nucleation and growth of the NAC-capped QDs. As shown in Fig. $3 \mathrm{~d}$, the peaks centered around $162 \mathrm{eV}$ were attributed to the S2p spectra. ${ }^{18,34}$ Three CdHgTe/CdS/CdZnS samples with different thiol ligands were examined, and their corresponding S2p spectra were separated into a doublet structure. Owing to the spin-orbit splitting, a doublet structure indicated that two types of $\mathrm{S}$ atoms corresponding to the $\mathrm{S} 2 \mathrm{p}_{1 / 2}$ peaks at $162.7 \mathrm{eV}$ and $S 2 p_{3 / 2}$ at $161.5 \mathrm{eV}$ were present. ${ }^{18}$ This finding demonstrates that the thiols not only served as a stabilizer for the QDs, but also underwent thermal decomposition to release free $\mathrm{S}^{2-} .^{\mathbf{1 8 , 3 4}}$ Ostensibly, for the MPA- and NAC-capped QDs, the intensity of $\mathrm{S}^{2-}$ as Cd-S was stronger than the intensity of $\mathrm{RS}^{-}$as Cd-SR. This suggested that the shell thickness of CdS or CdZnS depended on their epitaxial growth on the CdHgTe core. ${ }^{18}$ In comparison, the corresponding peaks of $\mathrm{Cd}-\mathrm{S}$ and $\mathrm{Cd}-\mathrm{SR}$ for TGA presented nearly the same content, thus suggesting that plenty of TGA acted as a stabilizer on the exterior of the QDs; this finding is consistent with the DLS results (Fig. 2a). Fourier Transform Infrared Spectroscopy (FT-IR) identified the representative functional groups of the as-prepared QDs. In Fig. S4, $\dagger$ the characteristic peaks at $2556 \mathrm{~cm}^{-1}$ correspond to the $\mathrm{S}-\mathrm{H}$ stretching vibration. ${ }^{\mathbf{1 0 , 3 5}}$ However, for the CdHgTe/CdS/CdZnS samples, the corresponding peaks disappeared (Fig. 3e), which indicated that a new bond was formed between the thiol ligands and the QDs. ${ }^{10,35}$ Furthermore, for the NAC-capped QDs, the peaks located at 2989 and $2931 \mathrm{~cm}^{-1}$ are due to the characteristic absorptions of methyl and methylene. ${ }^{10,35}$ The above data prove that the thiol group of NAC successfully bonded onto the surfaces of the QDs. 

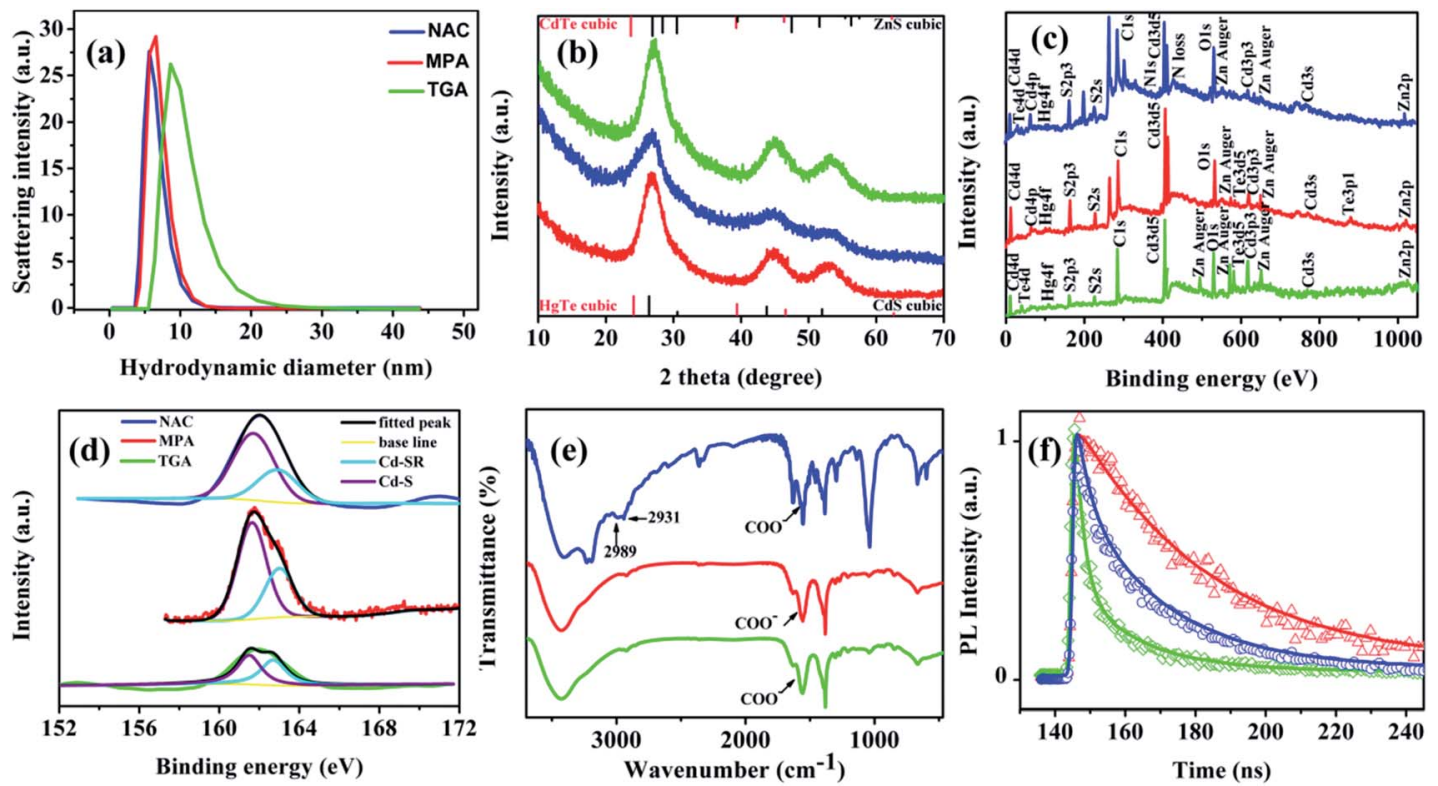

Fig. 3 DLS distributions (a), XRD patterns (b), XPS measurements (c), XPS narrow scan spectra of S2p spectra (d), FT-IR absorption spectra (e) and fluorescence decay curves (f) of CdHgTe/CdS/CdZnS QDs capped by NAC (blue), MPA (red) and TGA (green).

As seen clearly in Fig. 3f, the fluorescence decay curves of the samples reveal that the contribution of the non-radiative processes was significantly low because of the overgrowth of multiple shells. ${ }^{16,36}$

The variation in the fluorescence decay lifetimes was strongly dependent on the surface chemistry of the asprepared QDs. ${ }^{16,36}$ The average lifetime for the MPA-capped $\mathrm{CdHgTe/CdS/CdZnS} \mathrm{QDs} \mathrm{(32.8} \mathrm{ns)} \mathrm{was} \mathrm{longer} \mathrm{than} \mathrm{that} \mathrm{of}$ the QDs capped by NAC (24.2 ns), which is consistent with the results from the UV-PL spectra (Fig. 1). Previous studies on MPA-stabilized CdHgTe QDs demonstrated that the ligand shell was stable. ${ }^{27}$ Thus, the surface conditions were more ideal for the MPA- than the NAC-capped QDs. For TGA-capped QDs, more quenching defects on the surface of the shell were formed, thus resulting in reduced PLQYs and shortened average lifetimes (12.6 ns). ${ }^{\mathbf{1 6 , 3 6}}$

\section{In vitro imaging}

One of the biggest advantages of core-shell-shell QDs is their reduced tendency to photobleach (Fig. S5†). ${ }^{31,32}$ The photostability of the obtained CdHgTe/CdS/CdZnS QDs in the intracellular environment effectively exhibited this feature. It can be clearly observed in Fig. 4 that the stained Hela cells showed red fluorescence after $20 \mathrm{~min}$ irradiation. However, the contour of the cells stained with TGA-capped CdHgTe/CdS/CdZnS QDs became difficult to distinguish after $40 \mathrm{~min}$ of UV irradiation. By contrast, the PL intensity of the cells stained with NACcapped CdHgTe/CdS/CdZnS QDs only decreased slightly, thus indicating the high photostability in the intracellular environment under UV irradiation, as the NAC thiol effectively protected the QDs against degradation from the surface oxides resulting from the continuous UV irradiation. ${ }^{12,20,37,38}$
As the constituents of quantum dots, such as cadmium or mercury, are toxic to many cells, harmful effects can be expected. ${ }^{\mathbf{1 2 , 3 2}}$ However, the release of toxic ions might be prevented if the hydrophilic shell or the double shells around the QDs are stable. ${ }^{12,32}$ To investigate whether the CdHgTe/CdS/CdZnS QDS are cytotoxic to mammalian cells, we observed the fluorescence microscopy images for morphological changes and performed MTT assays using Hela cells. In the MTT assays, the cells did not exhibit significant cell death at each concentration of the obtained QDs solution within $30 \mathrm{~min}$, thus implying that the CSS structure effectively enhanced the stability of the QDs (Fig. S6 $\dagger$ ). When the incubation period was prolonged to $48 \mathrm{~h}$, the cell viability was drastically decreased as the concentration of the CdHgTe/CdS/CdZnS QDs capped by MPA and TGA was increased. This result is consistent with the phenomenon illustrated in Fig. 5, in which the fluorescence microscopy images of the cells show significant morphological changes at high concentrations of QDs (Fig. 5b and c). In comparison, the cells retained their shapes after $24 \mathrm{~h}$ of incubation with NAC-capped CdHgTe/CdS/ CdZnS QDs, thereby indicating that the NAC-capped QDS present outstanding biocompatibility (Fig. 5a). Environmental radiation can catalyze oxidation on the quantum dot surface and consequently cause an excessive release of ROS. ${ }^{20,37,38}$ As an antioxidant drug, NAC exhibit bioactivity and anti-oxidant activity by replacing the oxidative stress-induced toxicity. ${ }^{20,37,38}$ Therefore, the NAC-capped CdHgTe/CdS/CdZnS QDs have high stability and low cytotoxicity in living cells.

\section{In vivo toxicity evaluation}

The as-prepared QDs were similar in size to some viruses and large proteins. ${ }^{39,40}$ Thus, it is important to determine whether the particles or their constituents induced an inflammatory 


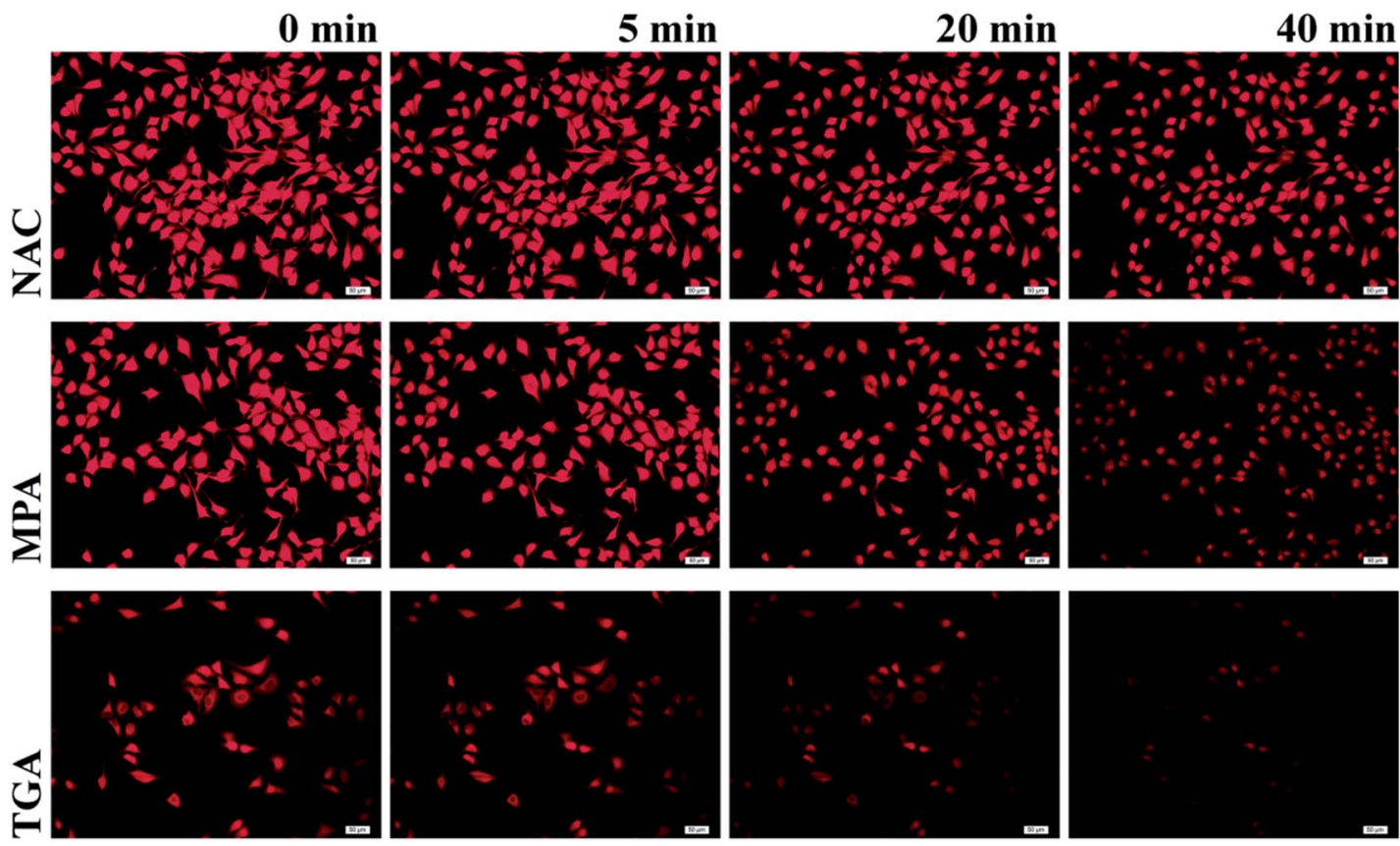

Fig. 4 Photostability experiments of $\mathrm{CdHgTe} / \mathrm{CdS} / \mathrm{CdZnS}$ QDs capped by NAC (top), MPA (middle) and TGA (bottom) in the intracellular environment.

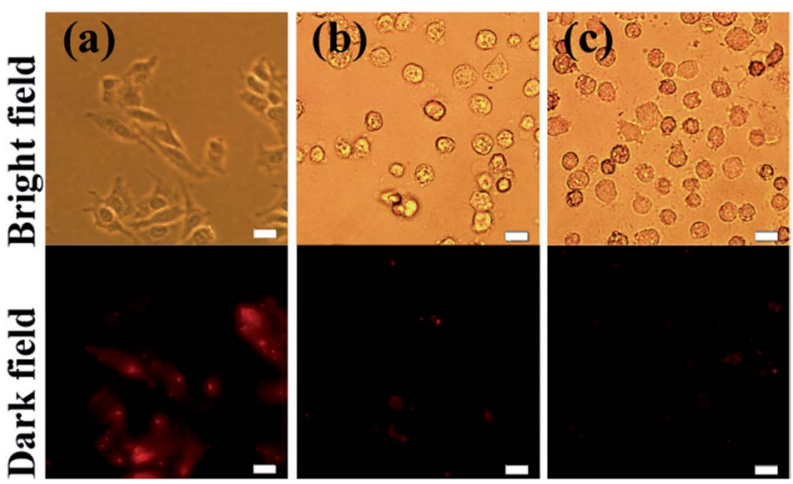

Fig. 5 Fluorescence microscopy images from live Hela cells labeled with CdHgTe/CdS/CdZnS QDs capped by NAC (a), MPA (b) and TGA (c) after $24 \mathrm{~h}$ incubation. Scale bars are $20 \mu \mathrm{m}$.

response and toxicity. ${ }^{\mathbf{1 4 , 1 5 , 2 1 , 3 9}}$ Body weights were monitored every day, and the fluctuations were negligible in the test and control groups (Fig. S7†). Furthermore, the mice's general behavior was monitored every day. No abnormalities in eating, drinking, grooming, activity, behavior and neurological status were observed. To continue the toxicity investigations, histological analyses from three treated mice and one control mouse were conducted to determine whether the QDs or their ligands caused tissue damage (Fig. 6). Two clinical pathologists analyzed the tissue sections and found no abnormality in the heart, lung and spleen. However, changes were observed in the kidneys and livers of mice treated with TGA- or MPA-capped QDs. Mice treated with TGA-capped QDs manifested a focal infiltration of congestion in some areas of the kidney and liver. As for MPA-capped QDs, a few congestions in the kidney as well as cellular swelling in the liver were observed. Overall, the NACcapped QDs displayed better biocompatibility than the QDS capped by MPA and TGA (Fig. S8 $\dagger$ ).

Histology provides macroscopic, visual and qualitative measurements of toxicity but is not quantitative. ${ }^{40}$ When the QDs were intravenously administered, the first physiological system they interacted with was the blood and the systema lymphaticum. ${ }^{15,21,39}$ In this case, the degradation of QDs or the presence of inappropriate surface coatings may induce an inflammatory response such as a change in white blood cell count. ${ }^{\mathbf{4 0 , 4 1}}$ The CSS QDs, which were coated with a biocompatible ligand, did not induce an inflammatory response (Fig. 7).

However, a slight inflammatory response of the blood components and a slight toxic effect on the bone marrow were observed, as revealed by the small change in the white blood cell count and hemoglobin concentration for the TGA- or MPAcapped QDs. ${ }^{39-41}$ This phenomenon may be attributed to the degradation of the surface coatings. ${ }^{39-41}$ The remaining hematology parameters are shown in Fig. S9. $\uparrow$ We further analyzed several major biochemistry results to assess whether the QDS induced toxicity to the liver and kidney functions (Fig. 7). The ALT, AST and CRE levels slightly increased for the TGA- or MPAcapped QDs. However, all the factors were within normal range. These results verified the absence of significant toxicity with respect to the control animals and reflected the normal variation present in the animal population. ${ }^{39-41}$ The remaining biochemistry parameters are shown in Fig. S10.†

\section{In vivo tumor targeting study}

Fig. 8 shows the fluorescence images after the as-prepared QDS were administered to the tumor-bearing mice. The dynamic 


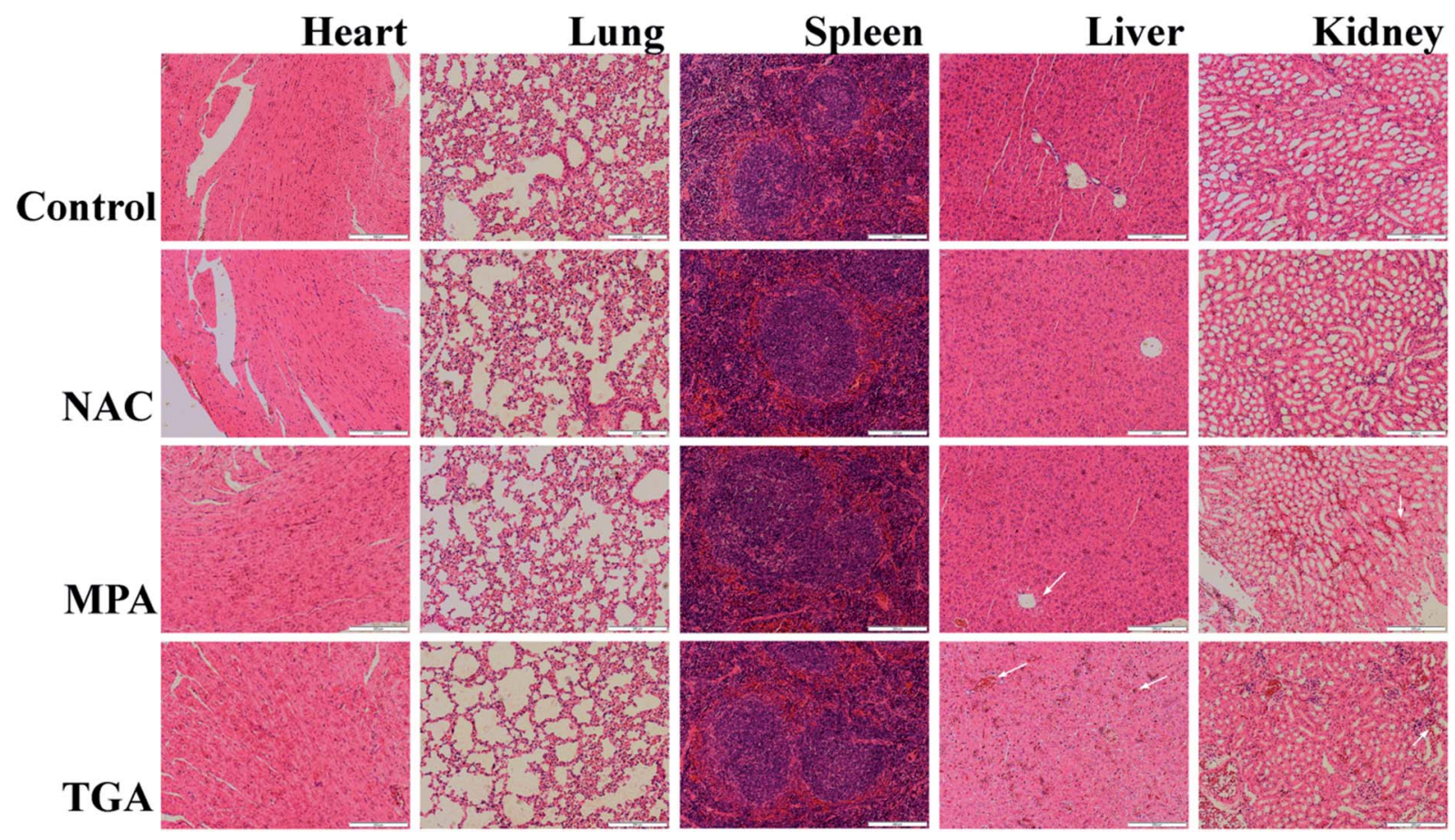

Fig. 6 Histological studies on the major organs of the CdHgTe/CdS/CdZnS QDs injected mice after 14 days. Scale bars are $200 \mu$ m.

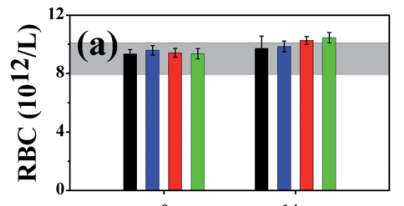

$\stackrel{0}{\text { Day after injection }}$
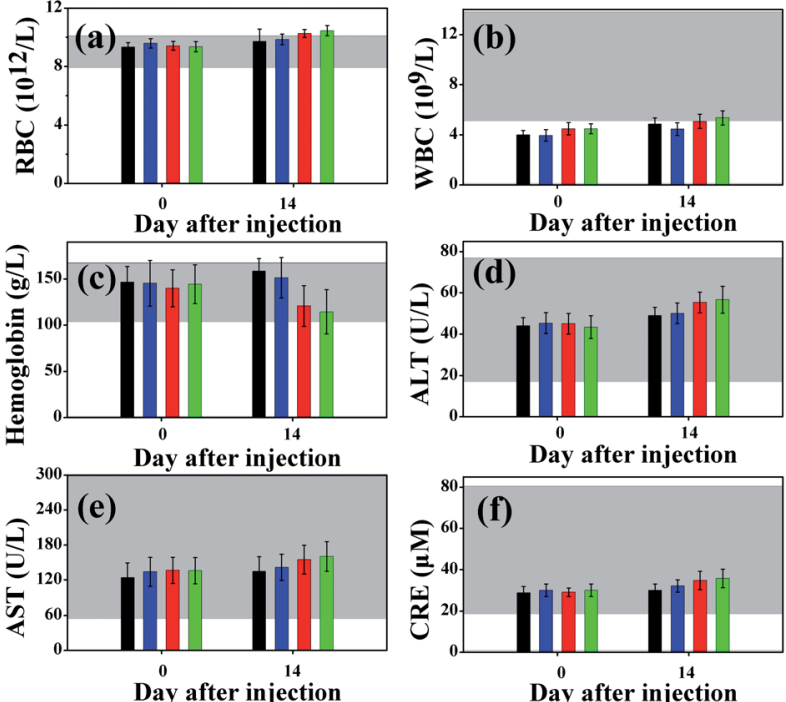

Day after injection
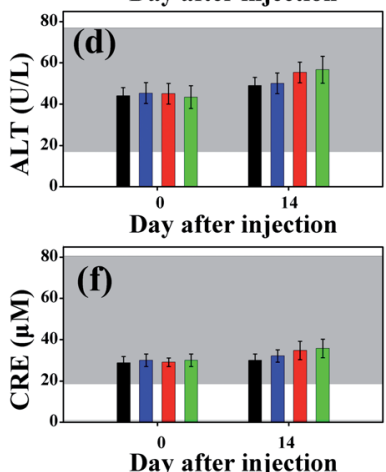

Fig. 7 Hematology and biochemistry results from mice treated with QDs of varying surface chemistry (NAC (blue), MPA (red) and TGA (green) capped $\mathrm{CdHgTe} / \mathrm{CdS} / \mathrm{CdZnS}$ QDs) or a vehicle control (black). (a-f) Mean and standard deviation of red blood cells, RBC (a), white blood cells, WBC (b), hemoglobin (c), alanine aminotransferase, ALT (d), aspartate aminotransferase, AST (e), creatinine, CRE (f). Grey bars indicate the range of values obtained from healthy BALB/c nude mice, as reported by Singh. ${ }^{41}$

properties of the NAC-capped QDs in the tumor-bearing mice completely differed from those in the mice treated with MPAand TGA-capped QDs. In general, active targeting (e.g. QDs conjugated with folic acid or hyaluronic acid is considerably faster and more efficient than passive acid) is considerably faster and more efficient than passive targeting. ${ }^{\text {42-44 }}$ However, the hydrodynamic diameter (HD) is always increased by crosslinking with the targeting ligands, thereby increasing the difficulty of renal clearance. ${ }^{45,46}$ Notably, the fluorescent signal of the NAC- capped QDs were rapidly concentrated on the tumor in only $0.5 \mathrm{~h}$, which was considerably faster than reported by Xue et al. $^{45}$ This phenomenon indicates that the ultra-small (HD $\leq 5$ $\mathrm{nm}$ ) and biocompatible QDs can migrate faster and penetrate in tissue more easily. ${ }^{22,42}$ By virtue of the leaky nature of tumor blood vessels, the QDs showed excellent accumulation in the tumor $2 \mathrm{~h}$ after injection (Fig. 8). Compared with the signal in the mice treated with NAC- or MPA-capped QDs, the fluorescence in the mouse treated with TGA-capped QDs was much weaker. The TGA-capped QDs have comparatively lower PLQYs, and the signal was much weaker upon penetrating tumor tissues. ${ }^{\mathbf{6}, 42,47}$ Nevertheless, the tumor tissue still yields a signal after $8 \mathrm{~h}$ when treated with TGA- or MPA-capped QDs. As we can see from Fig. 8a, the fluorescence signal sharply decreases after the injection of QDs for $8 \mathrm{~h}$. In general, the longer the nanoparticle circulation time in a living body, the greater the EPRinduced accumulation. ${ }^{43}$ This result suggested that the NACcapped QDs inside the body were easier to metabolize. ${ }^{22,45}$ The images of the ex vivo heart, liver, spleen, lung, bladder and kidney also proofed this viewpoint (Fig. 8d). In Fig. 8d, the FL intensity of the kidney and the bladder were notably stronger than those of other organs, indicating that renal clearance is the major elimination pathway for the ultra-small QDs. ${ }^{22,39,42,48}$ By contrast, the fluorescence of the liver and the spleen were evident in Fig. 8e and f, suggesting that most of the QDs slowly degraded and were cleared through the biliary pathway. ${ }^{22,39,42,49}$ 

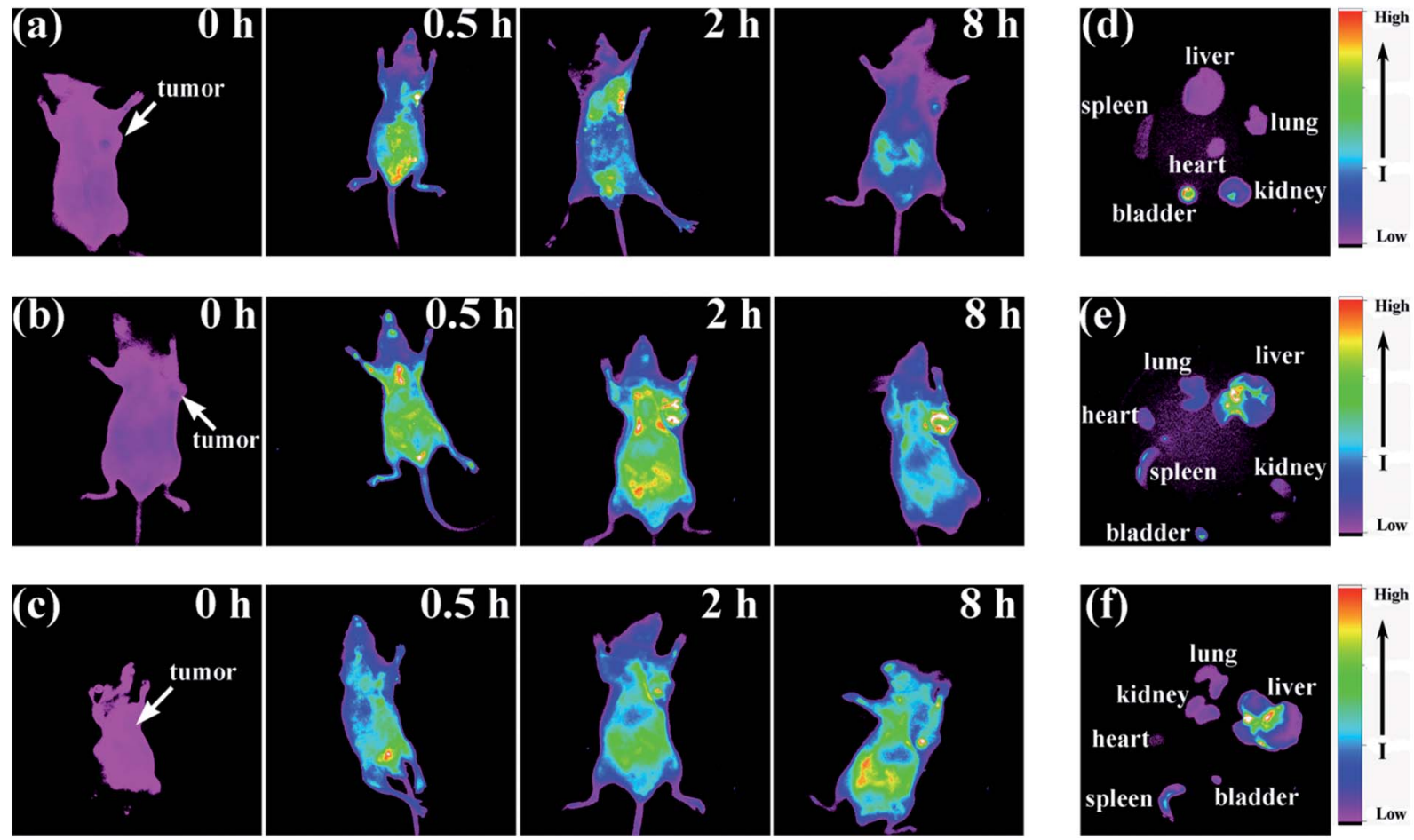

Fig. 8 In vivo tumor-targeting capability of $\mathrm{CdHgTe/CdS/CdZnS} \mathrm{QDs} \mathrm{capped} \mathrm{by} \mathrm{NAC} \mathrm{(a),} \mathrm{MPA} \mathrm{(b)} \mathrm{and} \mathrm{TGA} \mathrm{(c).} \mathrm{Fluorescence} \mathrm{images} \mathrm{of} \mathrm{ex} \mathrm{vivo}$ heart, liver, spleen, lung and kidney after injecting the as-prepared QDs capped by NAC (d), MPA (e) and TGA (f) for 8 h.

\section{Conclusion}

Herein, we demonstrated a one-pot aqueous synthesis approach to produce high PLQYs, NIR-emitting CdHgTe/CdS/ CdZnS core-shell-shell QDs. The use of different ligands (NAC, MPA and TGA) resulted in varied growth mechanisms, optical properties, HD, surface chemistry, toxicity and tumortargeting capabilities. Although TGA was a suitable ligand for fabricating long-wavelength-emission $\mathrm{CdHgTe} / \mathrm{CdS} / \mathrm{CdZnS}$ QDs, the NAC- and MPA-capped QDs yielded high PLQYs and preferred photostability. Notably, the NAC-capped QDs displayed the smallest hydrodynamic diameter and an excellent oxidation resistance in aqueous solutions and intracellular environments. When the concentration of the obtained QDs was increased and the incubation period was prolonged, the NAC-capped QDs exhibited lower cytotoxicity and higher biocompatibility than the MPA- and TGA-capped QDs. In vivo toxicity measurement revealed that the programmed NIRemitting QDs caused negligible harmful effects to nude mice even at a concentration of $20 \mathrm{mg} \mathrm{kg}^{-1}$. Compared with the TGA- and MPA-capped QDs with a large HD, the NAC-capped QDs offer a greater potential as a sensitive, efficient and fast tumor-targeting label. Furthermore, the QDs with ultra-small size resulted in rapid metabolism through renal filtration and urine excretion. By contrast, the TGA- and MPA-capped QDs remained in the vasculature for long periods of time. Therefore, the NAC-capped QDs are not limited to future medical imaging but have a promising prospect in basic life sciences.

\section{Conflicts of interest}

There are no conflicts to declare.

\section{Acknowledgements}

We acknowledge the financial support from the National Natural Science Foundation of China (No. 20577036, 20777058, 20977070, 81471789), the National Natural Science Foundation of Hubei province in China (No. 2015CFA137) and the National High Technology Research and Development Program of China (863 program, No. 2007AA06Z418). We express our gratitude to Zijian Wang and Dr Yanteng Zhao (Department of Biomedical Engineering, School of Basic Medical Sciences, Wuhan University), respectively, for technical help.

\section{References}

1 J. Shi, P. W. Kantoff, R. Wooster and O. C. Farokhzad, Nat. Rev. Cancer, 2017, 17, 20-37.

2 U. E. Martinezoutschoorn, M. Peirispagès, R. G. Pestell, F. Sotgia and M. P. Lisanti, Nat. Rev. Clin. Oncol., 2017, 14, 11-31.

3 D. S. Chen and I. Mellman, Science, 2017, 541, 321-330.

4 J. Michaelson, S. Satija, R. Moore, G. Weber, E. Halpern, A. Garland, D. B. Kopans and K. Hughes, Journal of Women s Imaging, 2003, 5, 3-10.

5 R. G. Aswathy, Y. Yoshida, T. Maekawa and D. S. Kumar, Anal. Bioanal. Chem., 2010, 397, 1417-1435. 
6 Q. Ma and X. G. Su, Analyst, 2010, 135, 1867-1877.

7 H. Kobayashi, M. Ogawa, R. Alford, P. L. Choyke and Y. Urano, Chem. Rev., 2010, 110, 2620-2640.

8 Z. Li, Q. Sun, Y. Zhu, B. Tan, Z. P. Xu and S. X. Dou, J. Mater. Chem. B, 2014, 2, 2793-2818.

9 H. Sun, H. Zhang, J. Ju, J. Zhang, G. Qian, C. Wang, B. Yang and Z. Y. Wang, Chem. Mater., 2008, 20, 6764-6769.

10 Q. Q. Wang, X. Y. Yu, G. Q. Zhan and C. Y. Li, Biosens. Bioelectron., 2014, 54, 311-316.

11 J. M. Tsay, M. Pflughoefft, L. A. Bentolila and S. Weiss, J. Am. Chem. Soc., 2004, 126, 1926-1927.

12 X. J. Liu, P. J. Zhou, H. J. Zhan, H. Y. Liu, J. W. Zhang and Y. N. Zhao, RSC Adv., 2017, 7, 29998-30007.

13 M. B. Yatvin, W. Kreutz, B. A. Horwitz and M. Shinitzky, Science, 1980, 210, 1253-1255.

14 Z. L. Cheng, A. A. Zaki, J. Z. Hui, V. R. Muzykantov and A. Tsourkas, Science, 2012, 338, 903-910.

15 T. S. Hauck, R. E. Anderson, H. C. Fischer, S. Newbigging and W. C. W. Chan, Small, 2010, 6, 138-144.

16 J. Wang, Y. T. Long, Y. L. Zhang, X. H. Zhong and L. Y. Zhu, ChemPhysChem, 2009, 10, 680-685.

17 Y. He, Y. L. Zhong, Y. Y. Su, Y. M. Lu, Z. Y. Jiang, F. Peng, T. T. Xu, S. Su, Q. Huang, C. H. Fan and S. T. Lee, Angew. Chem., Int. Ed., 2011, 50, 5695-5698.

18 H. B. Bao, Y. J. Gong, Z. Li and M. Y. Gao, Chem. Mater., 2004, 16, 3853-3859.

19 K. G. Li, J. T. Chen, S. S. Bai, X. Wen, S. Y. Song, Q. Yu, J. Li and Y. Q. Wang, Toxicol. in Vitro, 2009, 23, 1007-1013.

20 S. D. Flora, A. Izzotti, F. D'Agostini and R. M. Balansky, Carcinogenesis, 2001, 22, 999-1013.

21 H. S. Choi, W. H. Liu, F. B. Liu, K. Nasr, P. Misra, M. G. Bawendi and J. V. Frangioni, Nat. Nanotechnol., 2010, 5, 42-47.

22 H. S. Choi, W. Liu, P. Misra, E. Tanaka, J. P. Zimmer, B. I. Ipe, M. G. Bawendi and J. V. Frangioni, Nat. Biotechnol., 2007, 25, 1165-1170.

23 M. X. Yu and J. Zheng, ACS Nano, 2015, 9, 6655-6674.

24 X. J. Song, H. Gong, S. N. Yin, L. Cheng, C. Wang, Z. W. Li, Y. G. Li, X. Y. Wang, G. Liu and Z. Liu, Adv. Funct. Mater., 2014, 24, 1194-1201.

25 W. W. Yu, L. Qu, W. Guo and X. Peng, Chem. Mater., 2003, 15, 2854-2860.

26 P. Reiss, M. Protière and L. Li, Small, 2009, 5, 154-168.

27 S. Leubner, R. Schneider, A. Dubavik, S. Hatami, N. Gaponik, U. Resch-Genger and A. Eychmüller, J. Mater. Chem. C, 2014, 2, 5011-5018.

28 Y. He, L. M. Sai, H. T. Lu, M. Hu, W. Y. Lai, Q. L. Fan, L. H. Wang and W. Huang, Chem. Mater., 2007, 19, 359-365.

29 B. Tang, F. Yang, Y. Lin, L. H. Zhuo, J. C. Ge and L. H. Cao, Chem. Mater., 2007, 19, 1212-1214.
30 H. F. Qian, C. Q. Dong, J. L. Peng, X. Qiu, Y. H. Xu and J. C. Ren, J. Phys. Chem. C, 2007, 111, 16852-16857.

31 H. J. Zhan, P. J. Zhou, K. L. Pan, T. He, X. He, C. Y. Zhou and Y. N. He, J. Nanopart. Res., 2013, 15, 1680-1692.

32 Y. He, H. T. Lu, L. M. Sai, Y. Y Su, M. Hu, C. H. Fan, W. Huang and L. H. Wang, Adv. Mater., 2008, 20, 3416-3421.

33 V. Lesnyak, A. Lutich, N. Gaponik, M. Grabolle, A. Plotnikov, U. Resch-Genger and A. Eychmüller, J. Mater. Chem., 2009, 19, 9147-9152.

34 Z. H. Sheng, H. Y. Han, X. F. Hu and C. Chi, Dalton Trans., 2010, 39, 7017-7020.

35 H. J. Zhan, P. J. Zhou, R. Ma, X. J. Liu, Y. N. He and C. Y. Zhou, J. Fluoresc., 2014, 24, 57-65.

36 Q. H. Zeng, X. G. Kong, Y. J. Sun, Y. L. Zhang, L. P. Tu, J. L. Zhao and H. Zhang, J. Phys. Chem. C, 2008, 112, 85878593.

37 W. J. Parak, T. Pellegrino and C. Plank, Nanotechnology, 2005, 16, 9-25.

38 Y. Y. Su, M. Hu, C. H. Fan, Y. He, Q. N. Li, W. X. Li, L. H. Wang, P. P. Shen and Q. Huang, Biomaterials, 2010, 31, 4829-4834.

39 H. S. Choi, B. I. Ipe, P. Misra, J. H. Lee, M. G. Bawendi and J. V. Frangioni, Nano Lett., 2009, 9, 2354-2359.

40 L. Ye, K. T. Yong, L. Liu, I. Roy, R. Hu, J. Zhu, H. Cai, W. C. Law, J. Liu, K. Wang, J. Liu, Y. Liu, Y. Hu, X. Zhang, M. T. Swihart and P. N. Prasad, Nat. Nanotechnol., 2012, 7, 453-458.

41 N. Singh, S. Charan, K. Sanjiv, S. H. Huang, Y. C. Hsiao, C. W. Kuo, F. C. Chien, T. C. Lee and P. Chen, Bioconjugate Chem., 2012, 23, 421-430.

42 Z. Li, Q. Sun, Y. Zhu, B. Tan, Z. P. Xu and S. X. Dou, J. Mater. Chem. B, 2014, 2, 2793-2818.

43 Z. L. Cheng, A. A. Zaki, J. Z. Hui, V. R. Muzykantov and A. Tsourkas, Science, 2012, 338, 903-910.

44 G. Hong, J. T. Robinson, Y. Zhang, S. Diao, A. L. Antaris, Q. Wang and H. Dai, Angew. Chem., Int. Ed., 2012, 124, 9956-9959.

45 B. Xue, D. W. Deng, J. Cao, F. Liu, X. Li, W. Akers, S. Achilefu and Y. Q. Gu, Dalton Trans., 2012, 41, 4935-4947.

46 Y. M. Lu, Y. L. Zhong, J. Wang, Y. Y. Su, F. Peng, Y. F. Zhou, X. X. Jiang and Y. He, Nanotechnology, 2013, 16, 135101135109.

47 P. G. Luo, F. Yang, S. T. Yang, S. K. Sonkar, L. J. Yang, J. J. Broglie, Y. Liu and Y. P. Sun, RSC Adv., 2014, 4, 1079110807.

48 X. Michalet, F. F. Pinaud, L. A. Bentolila, J. M. Tsay, S. Doose, J. J. Li, G. Sundaresan, A. M. Wu, S. S. Gambhir and S. Weiss, Science, 2005, 307, 538-544.

49 Y. Kong, J. Chen, H. Fang, G. Heath, Y. Wo, W. Wang, Y. Li, Y. Guo, S. D. Evans, S. Chen and D. Zhou, Chem. Mater., 2016, 28, 3041-3050. 